\title{
Quantum dissipative Higgs model
}

\author{
Ehsan Amooghorban*and Ali Mahdifar ${ }^{\dagger}$ \\ Department of Physics, Faculty of Basic Sciences, Shahrekord University, \\ P.O. Box 115, Shahrekord 88186-34141, Iran.
}

January 16, 2021

\begin{abstract}
By using a continuum of oscillators as a reservoir, we present a classical and a quantum-mechanical treatment for the Higgs model in the presence of dissipation. In this base, a fully canonical approach is used to quantize the damped particle on a spherical surface under the action of a conservative central force, the conjugate momentum is defined and the Hamiltonian is derived. The equations of motion for the canonical variables and in turn the Langevin equation are obtained. It is shown that the dynamics of the dissipative Higgs model is not only determined by a projected susceptibility tensor that obeys the Kramers-Kronig relations and a noise operator but also the curvature of the spherical space. Due to the gnomonic projection from the spherical space to the tangent plane, the projected susceptibility displays anisotropic character in the tangent plane. To illuminate the effect of dissipation on the Higgs model, the transition rate between energy levels of the particle on the sphere is calculated. It is seen that appreciable probabilities for transition are possible only if the transition and reservoir's oscillators frequencies to be nearly on resonance.
\end{abstract}

Keywords: Higgs model; Dissipation; Langevin equation; Susceptibly tensor.

PACS: 03.65.-w, 04.62.+v, 11.10.Ef, 05.40.Ca, 03.70.+k

\footnotetext{
*Ehsan.amooghorban@sci.sku.ac.ir

†'mahdifar_a@sci.sku.ac.ir
} 


\section{Introduction}

Higgs and Leemon investigated the non-relativistic motion of a particle embedded on a sphere under the influence of conservative central potentials [1, 2]. These central potentials reduce to the familiar Coulomb and isotropic oscillator potentials of a Euclidean geometry when the curvature of the sphere goes to zero. Higgs defined the motion on a sphere of a constant radius by means of a gnomonic projection from the motion of a plane, tangent to the sphere at a given point. The advantage of this projection over all others for the analysis of particle motion on a sphere derives from the fact that free particle motion, uniform motion on a great circle, projects into a rectilinear plane while its non-uniform motion projects into the tangent plane. In other words, the projected free particle orbits are the same as those in the Euclidean geometry: the curvature affects only the speed of the projected motion. Higgs also showed that this feature persists in the presence of a central force derived from a potential $\mathrm{V}(\mathrm{r})$, i.e. he proved that the dynamical symmetries in a sphere are the same as those in the plane. Using the Hamiltonians as functions of the Casimir operators, the eigenstates and eigenvalues of the two systems were also obtained in [1] and [2], respectively.

In Ref. [3], based on the Higgs model, we have constructed the generalized (nonlinear) coherent states [4] of a two-dimensional harmonic oscillator on a spherical surface to study some of their quantum optical properties. Accordingly, we have developed a feasible physical model to generate nonlinear coherent states on a sphere in a generalized trapped ion system [5]. The geometry and the algebra of the sphere coherent states is studied in [6, 7]. It has been shown that the structure and properties of sphere-(nonlinear) coherent states could be explored to studying the curvature effects of physical space on both transition probability and geometric phase.

Moreover, we have investigated a two-dimensional isotropic harmonic oscillator on a sphere with a time-dependent radius [8]. It is seen that variations in the sphere radius could be represented by a minimally coupled Hamiltonian. As a realization of the model, a two-dimensional isotropic harmonic oscillator was considered on a fluctuating background. A simple golden rule was obtained for the transition probabilities per unit time between energy levels.

However, the classical and the quantum-mechanical treatment of the Higgs model have been surveyed so far, are the limit of a more general formalism for the case where the dissipative effects is neglected. But, we know 
that the dissipation is a ubiquitous phenomenon in real physical systems and in turn leads to the remarkable effects for more physical systems. We therefore expect that the dissipative effects affect the dynamical properties of a quantum particle which is constrained to move on a spherical surface and lead to some interesting results. The question which naturally arises in this context, how can we describe the Higgs model in the presence of the dissipation? The present paper is intended to respond to this question.

On the other hand, in classical physics the dissipation effects can be described by introducing a velocity proportional force in the equation of motion. In quantum mechanics, we can borrow the idea of force from the classical dynamics. When the force is conservative, the formulation of the quantum analogue of the classical motion is straightforward. But, the inclusion of dissipation by introducing the velocity proportional force require a time dependent Lagrangian and Hamiltonian and this leads to the inconsistencies between the equations of motion and the canonical commutation relations. In addition, the result depends on our choice of the Lagrangian or the Hamiltonian. Several approaches have been developed to circumvent this problem [9]-[18]. The most successful and rather general approach is based on a reservoir concept by introducing it explicitly in the quantization process. Although, knowing the microscopic details of the reservoir is not necessarily needed.

Recently, we have presented a Lagrangian scheme to the quantization of the dissipative systems [19]. This approach allows us to obtain a deeper understanding from the mechanism of the dissipation in the higgs model, as well as it brings forth the simplest way in which the dissipation and the fluctuation effects emerge from the classical to the quantum relativistic domain. In the quantization process, it is seen that a reservoir containing of a continuum of three dimensional harmonic oscillators mimics the isotropic [20] and anisotropic dissipative materials [19] and even the nonlinear dissipative materials [21]. Consequently, the main system including the reservoir is described by a time-independent Lagrangian and Hamiltonian and the quantization of the dissipative system is performed in a completely standard fashion.

The paper is organized as follows. In Sec. 2, we propose a Lagrangian for the total system and investigate a classical treatment of a dissipative particle embedded on a spherical space by means of the gnomonic coordinate. In Sec. 3 , we use the Lagrangian introduced in the Sec. 2 to canonically quantize the system and obtain the corresponding Langevin equation. Subsequently, in Sec. 4, as a simple application, we calculate the transition rate between 
energy levels of the particle on the sphere and show that how the curvature of physical space and the dissipative effect compete in the transition rate of aforementioned particle. Finally, the summary and concluding remarks are given in section 5 .

\section{Classical dynamics}

The dissipative effects for more physical systems are important and can not be ignored. One of these interesting systems, which has been studied by Higgs [1], is a quantum particle on a two-dimensional sphere under the action of a conservative central force. In the higgs model, we may imagine that the dissipation effects introduce to our formalism by interacting the main system with the quantum vacuum field and/or a lossy medium. This approach based on the Lagrangian quantization scheme therefore prepares the grounds to extend the Higgs model to include the dissipative effects. In [19], the classical and the quantum description of a dissipative system was accomplished by modeling a system interacting with a reservoir. The reservoir was assumed there made up a continuum of three dimensional harmonic oscillators labeled by a continuous parameter $\omega$. This reservoir modeling in fact was inspired on the basis of the microscopic Hayfield model of a dielectric [22]. We apply this approach to examine the classical and the quantum treatment of the dissipative particle embedded on the spherical space. Let us start the following classical Lagrangian for the total system

$$
L(t)=L_{\mathrm{r}}(t)+L_{\mathrm{s}}(t)+L_{\mathrm{int}}(t) .
$$

The first term $L_{\mathrm{r}}(t)$ is the Lagrangian of the reservoir which is a continuum of three dimensional harmonic oscillators defined by

$$
L_{\mathrm{r}}(t)=\frac{1}{2} \int_{0}^{\infty} d \omega\left[\dot{X}_{i}^{2}(\omega, t)-\omega^{2} X_{i}^{2}(\omega, t)\right],
$$

where $X_{i}(\omega, t), i=1,2,3$, are the Cartesian components of the reservoir variable and the Einstein convention for repeated indices is used.

The second term $L_{\mathrm{s}}(t)$ is the Lagrangian of our main system on a sphere

with radius $R$ under the influence of a conservative centeral force derived from a potential $V(|\mathbf{q}|)$, given by

$$
L_{\mathrm{s}}(t)=\frac{1}{2} m \dot{\mathbf{q}}^{2}(t)-V(|\mathbf{q}|),
$$


where the Cartesian coordinates of the particle embedded on the spherical space are designated by $\mathbf{q}=\left(q_{1}, q_{2}, q_{3}\right)$ and is assumed that they satisfy the sphere equation: $q_{1}^{2}+q_{2}^{2}+q_{3}^{2}=\frac{1}{R^{2}}$.

In [1, 2], the authors introduced a gnomonic projection by means of the projection of a point on the sphere through its center onto the tangent plane in the embedding space. The advantage of this projection over all others for the analysis of the motion of a particle on a sphere stems from the fact that a free particle on a sphere just affects by the curvature of the sphere and the form of the central potentials does not change by the curvature of the sphere. We can use this advantage by applying the gnomonic projection to the Lagrangian of our system. To this end, we first express the relationship between the Cartesian coordinates $\mathbf{q}$ of the particle embedded on the sphere and two Cartesian coordinates $x_{\alpha}(\alpha=1,2)$ of the tangent plane as

$$
\mathbf{q}=\Lambda^{-1}\left(x_{1}, x_{2}, \lambda^{-\frac{1}{2}}\right)
$$

where $\lambda=R^{-2}$ is the curvature of the sphere, $\Lambda=\sqrt{1+\lambda r^{2}}$ and $r^{2}=x_{\beta} x_{\beta}$. On the other hand, for further calculations, it is convenient to obtain the metric tensor of the physical space. According to above relation, It can be easily shown that the metric tensor of physical space in terms of the tangent space coordinates is $\overline{\bar{g}}(\mathbf{x}, \lambda)=\Lambda^{-2}\left(\mathbf{1}-\lambda \Lambda^{-2} \mathbf{x x}\right)$. This enable us to rewrite the Lagrangian (3) in term of the new variable $x_{\alpha}$ as

$$
L_{\mathrm{s}}(t)=\frac{1}{2 \Lambda^{2}}\left(\dot{\mathbf{x}}^{2}-\lambda \frac{(\mathbf{x} \cdot \dot{\mathbf{x}})^{2}}{\Lambda^{2}}\right)-V(\mathbf{x}) .
$$

The Lagrangian $L_{\text {int }}(t)$ is the interaction term defined by

$$
L_{\mathrm{int}}(t)=\int_{0}^{\infty} d \omega f(\omega) \dot{q}_{i}(t) X_{i}(\omega, t)
$$

Similar to the main system Lagrangian (5), we can rewrite $L_{\text {int }}(t)$ in term of the coordinate of the tangent plane as

$$
L_{\text {int }}(t)=\int_{0}^{\infty} d \omega f(\omega) \overline{\bar{a}}_{i \beta}(\mathbf{x}, \lambda) \dot{x}_{\beta} X_{i}(\omega, t)
$$

where the geometric tensor $\overline{\bar{a}}(\mathbf{x}, \lambda)$ is defined in term of the metric tensor as $\overline{\bar{a}}_{i \alpha} \overline{\bar{a}}_{i \beta}(\mathbf{x}, \lambda)=\overline{\bar{g}}_{\alpha \beta}(\mathbf{x}, \lambda)$. Now, the total Lagrangian (1) has been specified in term of the coordinates of the tangent plane, we can use the Euler-Lagrange 
equation to obtain the classical equations of motion. We find that the classical equations for dynamical variables $\mathbf{x}$ of the spherical particle and $\mathbf{X}(\omega, t)$ of the reservoir, are respectively

$$
\ddot{x}_{\alpha}-\frac{2 \lambda(\mathbf{x} \cdot \dot{\mathbf{x}})}{\Lambda^{2}} \dot{x}_{\alpha}+\frac{\Lambda^{4}}{r} \frac{d V(r)}{d r} x_{\alpha}=-\overline{\bar{g}}_{\alpha \beta}^{-1} \dot{R}_{\beta},
$$

and

$$
\ddot{X}_{i}(\omega, t)+\omega^{2} X_{i}(\omega, t)=\overline{\bar{a}}_{i \beta}(\mathbf{x}, \lambda) \dot{x}_{\beta}(t) f(\omega),
$$

where $\overline{\bar{g}}^{-1}(\mathbf{x}, \lambda)=\Lambda^{2}(\mathbf{1}+\lambda \mathbf{x x})$ is the inverse of the metric tensor $\overline{\bar{g}}$, and the components of the field $\mathbf{R}$ are defined by

$$
R_{\alpha}(\mathbf{x}, \lambda ; t)=\overline{\bar{a}}_{j \alpha}(\mathbf{x}, \lambda) \int_{0}^{\infty} d \omega f(\omega) X_{j}(\omega, t)
$$

The formal solution of the field equation (9) is obtained as

$$
\begin{aligned}
X_{i}(\omega, t)= & \dot{X}_{i}(\omega, 0) \frac{\sin \omega t}{\omega}+X_{i}(\omega, 0) \cos \omega t \\
& +\overline{\bar{a}}_{i \beta}(\mathbf{x}, \lambda) \int_{0}^{t} d t^{\prime} \frac{\sin \omega\left(t-t^{\prime}\right)}{\omega} f(\omega) \dot{x}_{\beta}\left(t^{\prime}\right)
\end{aligned}
$$

where the two first term is associated with the homogeneous solution of Eq. (9) and the third term is related to the inhomogeneous one. By substituting the solution $X_{i}(\omega, t)$ into the Eq. (10), the field $\mathbf{R}$ is obtained as

$$
R_{\alpha}(\mathbf{x}, \lambda ; t)=\int_{0}^{\infty} d t^{\prime} \overline{\bar{\chi}}_{\alpha \beta}\left(\mathbf{x}, \lambda ; t-t^{\prime}\right) \dot{x}_{\beta}\left(t^{\prime}\right)+R_{\alpha}^{\mathrm{N}}(\mathbf{x}, \lambda ; t) .
$$

The medium susceptibility tensor projected on the tangent space, $\overline{\bar{\chi}}_{\alpha \beta}(\mathbf{x}, \lambda ; t)$, is given by

$$
\overline{\bar{\chi}}_{\alpha \beta}(\mathbf{x}, \lambda ; t)=\overline{\bar{g}}_{\alpha \beta}(\mathbf{x}, \lambda) \gamma(t),
$$

where $\gamma(t)=\int_{0}^{\infty} d \omega f(\omega) \frac{\sin \omega t}{\omega} \Theta(\mathrm{t})$ is the medium susceptibility function felt by the particle on the sphere. Incidentally, this relation shows that the susceptibility function $\gamma$ and in turn the projected susceptibility tensor $\overline{\bar{\chi}}$ satisfy the significant Kramers-Kronig relations, as they must be. Mathematically, these two susceptibility tensors are related to each other via the metric tensor. 
Therefore, we except that, even though the medium susceptibility function $\gamma(t)$ is isotropic and homogenous for the particle on the sphere, the projected susceptibility tensor on the tangent plane, $\overline{\bar{\chi}}$, is not only anisotropic and inhomogeneous but also depends on the curvature of the sphere. This is not surprising, because even in the absence of the dissipative effects, the free particle motion on a sphere is projected onto rectilinear, but non-uniform motion on the tangent plane [1.

Given an arbitrary susceptibility function $\gamma$, the coupling tensor $f(\omega)$ can be uniquely determined. By taking the inversion of Eq. (13), the corresponding coupling function is obtained as following:

$$
\begin{aligned}
f(\omega) & =\sqrt{\frac{2 \omega}{\pi} \overline{\bar{g}}_{\alpha \beta}^{-1}(\mathbf{x}, \lambda) \operatorname{Im}\left[\overline{\bar{\chi}}_{\alpha \beta}(\mathbf{x}, \lambda ; \omega)\right]} \\
& =\sqrt{\frac{2 \omega}{\pi} \operatorname{Im}[\gamma(\omega)]},
\end{aligned}
$$

where $\gamma(\omega)$ is defined as

$$
\begin{aligned}
\gamma(\omega) & =\int_{0}^{\infty} d t \gamma(t) e^{\imath \omega t} \\
& =\int_{0}^{\infty} d \omega^{\prime} \frac{f^{2}(\omega)}{\omega^{\prime 2}-\omega^{2}+\imath 0^{+}} .
\end{aligned}
$$

The second term in Eq. (12) are components of a noise vector which is defined in terms of the medium susceptibility function $\gamma(t)$ and the geometry tensor $\overline{\bar{a}}(\mathbf{x}, \lambda)$ as

$$
\begin{aligned}
R_{\alpha}^{\mathrm{N}}(\mathbf{x}, \lambda ; t)= & \left.\overline{\bar{a}}_{\alpha j}(\mathbf{x}, \lambda) \int_{0}^{\infty} d \omega \sqrt{\frac{2 \omega}{\pi} \operatorname{Im}[\gamma(\omega)}\right] \\
& \times\left(\dot{X}_{j}(\omega, 0) \frac{\sin \omega t}{\omega}+X_{j}(\omega, 0) \cos \omega t\right) .
\end{aligned}
$$

In this manner, the field $\mathbf{R}(\mathbf{x}, \lambda ; t)$ is completely determined in term of the medium susceptibility function $\gamma(t)$, the geometry tensor $\overline{\bar{a}}(\mathbf{x}, \lambda)$ and the noise vector $\mathbf{R}^{\mathrm{N}}(\mathbf{x}, \lambda ; t)$. Now, by inserting Eq. (12) into Eq. (9), we obtain the classical Langevin equation as

$$
\ddot{x}_{\alpha}-\frac{2 \lambda(\mathbf{x} \cdot \dot{\mathbf{x}})}{\Lambda^{2}} \dot{x}_{\alpha}+\int_{0}^{\infty} d t^{\prime} \dot{\gamma}\left(t-t^{\prime}\right) \dot{x}_{\alpha}\left(t^{\prime}\right)+\frac{\Lambda^{4}}{r} \frac{d V(r)}{d r} x_{\alpha}=\xi^{\mathrm{N}}(\mathbf{x}, \lambda ; t),
$$


where the stochastic force induced by the reservoir, $\xi^{\mathrm{N}}(\mathbf{x}, \lambda ; t)$, is given by

$$
\xi^{\mathrm{N}}(\mathbf{x}, \lambda ; t)=-\overline{\bar{g}}_{\alpha \beta}^{-1}(\mathbf{x}, \lambda) \dot{R}_{\beta}^{\mathrm{N}}(\mathbf{x}, \lambda ; t)
$$

It is clear from Eq. (17) that the dissipative effects appear as a frictional force comprising the linear functional of the history of the velocity of the particle on the sphere and the inhomogeneous stochastic force $\xi^{\mathrm{N}}(\mathbf{x}, \lambda ; t)$. This conclusion is expected by referring to the consideration of Ref. [19], of course with a difference that the metric and the curvature of the sphere are arrived to our computation. In the limit $\gamma \rightarrow 0$, the classical Langevin equation (17) smoothly tend to the non-dissipative one which is indeed consistent with the result reported in [1].

\section{Quantum dynamics of the dissipative Higgs model}

By using the Lagrangian (11), we can now calculate canonical conjugate momentum associated with the dynamical variables $\mathbf{x}(t)$ and $\mathbf{X}(\omega, t)$, respectively, as

$$
\begin{aligned}
& \mathbf{p}(t)=\frac{\partial L}{\partial \dot{\mathbf{x}}}=\Lambda^{-2} \dot{\mathbf{x}}-\Lambda^{-4}(\mathbf{x} \cdot \dot{\mathbf{x}}) \dot{\mathbf{x}}+\mathbf{R}, \\
& \mathbf{P}(\omega, t)=\frac{\delta L}{\delta \dot{\mathbf{X}}(\omega, t)}=\dot{\mathbf{X}}(\omega, t)
\end{aligned}
$$

To consider the quantum dissipative Higgs model, we impose equal-time com-

mutation relations among the variables and the corresponding conjugates momentum as

$$
\begin{gathered}
{\left[x_{\alpha}(t), p_{\beta}(t)\right]=\iota \hbar \delta_{\alpha \beta},} \\
{\left[X_{i}(\omega, t), P_{j}\left(\omega^{\prime}, t\right)\right]=\iota \hbar \delta_{i j} \delta\left(\omega-\omega^{\prime}\right) .}
\end{gathered}
$$

Therefore, by using the Lagrangian (11) and the relations for the canonical conjugate variables in (21) and (22), we obtain the quantum Hamiltonian of the total system as

$$
\mathcal{H}=\mathcal{H}_{\text {Higgs }}(\mathbf{x}, \mathbf{p}-\mathbf{R} ; \lambda)+\frac{1}{2} \int_{0}^{\infty} d \omega\left(\mathbf{P}^{2}(\omega, t)+\omega^{2} \mathbf{X}^{2}(\omega, t)\right),
$$


where

$$
\mathcal{H}_{\text {Higgs }}(\mathbf{x}, \mathbf{p} ; \lambda)=\frac{1}{2}\left(\pi_{\alpha} \pi_{\alpha}+\frac{\lambda}{2} L_{\alpha \beta} L_{\alpha \beta}\right)+V(\mathbf{x})
$$

and $\boldsymbol{\pi}=\mathbf{p}+\frac{\lambda}{2}[\mathbf{x}(\mathbf{x} \cdot \mathbf{p})+(\mathbf{x} \cdot \mathbf{p}) \mathbf{x}]$. The above Hamiltonians (23) $-(24)$, together with the commutation relations (21)-(22), complete our procedure to describe the dissipative Higgs model as quantum mechanically. It is important to note that the Hamiltonian of the main system $\mathcal{H}_{\text {Higgs }}$ has the same form of the Higgs Hamiltonian [1], but with a difference that the momentum variable $\mathbf{p}$ is replaced by the expression $(\mathbf{p}-\mathbf{R})$. Indeed, this result justify the minimal coupling scheme was presented in [19] to introduce the dissipation effects into our formalism.

To facilitate our calculations, let us introduce the annihilation operator

$$
b_{i}(\omega, t)=\frac{1}{\sqrt{2 \hbar \omega}}\left[\omega X_{i}(\omega, t)+\imath P_{i}(\omega, t)\right] .
$$

By using the canonical commutation relations (21) and (21), we readily obtain

$$
\left[b_{i}(\omega, t), b_{j}^{\dagger}\left(\omega^{\prime}, t\right)\right]=\delta_{i j} \delta\left(\omega-\omega^{\prime}\right)
$$

with all other equal-time commutators being zero. By inverting Eq. (25), we can express the canonical conjugate variables $X_{i}(\omega, t)$ and $P_{i}(\omega, t)$ in terms of the creation and annihilation $b_{i}^{\dagger}(\omega, t)$ and $b_{i}(\omega, t)$. By inserting these relations into the Hamiltonian (23), we obtain the Hamiltonian of the total system as follows:

$$
\mathcal{H}=\mathcal{H}_{\text {Higgs }}(\mathbf{x}, \mathbf{p}-\mathbf{R} ; \lambda)+\mathcal{H}_{r}
$$

where

$$
R_{\alpha}(\mathbf{x}, \lambda)=\overline{\bar{a}}_{j \alpha}(\mathbf{x}, \lambda) \int_{0}^{\infty} d \omega \sqrt{\frac{\hbar}{\pi} \operatorname{Im}[\gamma(\omega)]}\left[b_{j}(\omega, t)+b_{j}^{\dagger}(\omega, t)\right],
$$

and

$$
\mathcal{H}_{r}=: \int d \omega \hbar \omega b_{j}^{\dagger}(\omega, t) b_{j}(\omega, t):
$$


is the Hamiltonian of the reservoir in normal ordering form. To study the dynamics of the dissipative particle on the sphere under the central potential $V(\mathbf{q})$, let us proceed in the Heisenberg picture. By using commutation relations (21), (22) and the total Hamiltonian (23), the equations of motion for the canonical variables $\mathbf{X}$ and $\mathbf{P}$ are obtained, respectively, as

$$
\begin{gathered}
\dot{X}_{i}(\omega, t)=\frac{\iota}{\hbar}\left[\mathcal{H}, X_{i}(\omega, t)\right]=P_{i}(\omega, t), \\
\dot{P}_{i}(\omega, t)=\frac{\iota}{\hbar}\left[\mathcal{H}, P_{i}(\omega, t)\right]=-\omega^{2} X_{i}(\omega, t)+f(\omega) \overline{\bar{a}}_{i \beta} \dot{x}_{\beta} .
\end{gathered}
$$

It can be easily shown that the combination of these equations are identical to the classical equation (9) with the formal solution (11). Calculations analogous to those of (30) and (31) give the following Heisenberg equations for the dynamical variables $\mathbf{x}$ and $\mathbf{p}$, respectively, as

$$
\begin{gathered}
\dot{\mathbf{x}}=\frac{\iota}{\hbar}[\mathcal{H}, \mathbf{q}(t)]=\Lambda^{2}(\mathbf{1}+\lambda \mathbf{x x}) \cdot(\mathbf{p}-\mathbf{R}) \\
\dot{\mathbf{p}}=\frac{\iota}{\hbar}[\mathcal{H}, \mathbf{p}(t)]=-\frac{\lambda\left(\mathbf{x} \dot{x}^{2}+\dot{x}(\mathbf{x} \cdot \dot{\mathbf{x}})\right)}{\Lambda^{4}}+\frac{2 \lambda^{2} \mathbf{x}(\mathbf{x} \cdot \dot{\mathbf{x}})^{2}}{\Lambda^{6}}-\nabla V(\mathbf{x})
\end{gathered}
$$

By combining the recent Heisenberg equations and eliminating the reservoir degrees of freedom by inserting the solution (11) into Eq. (32), we obtain the quantum analogous of the Langevin equation (17) as

$$
\ddot{x}_{\alpha}-\frac{2 \lambda(\mathbf{x} \cdot \dot{\mathbf{x}})}{\Lambda^{2}} \dot{x}_{\alpha}+\int_{0}^{\infty} d t^{\prime} \dot{\gamma}\left(t-t^{\prime}\right) \dot{x}_{\alpha}\left(t^{\prime}\right)+\frac{\Lambda^{4}}{r} \frac{d V(r)}{d r} x_{\alpha}=\xi^{\mathrm{N}}(\mathbf{x}, \lambda ; t),
$$

where the explicit form of the noise operator $\xi^{\mathrm{N}}(\mathbf{x}, \lambda ; t)$ is written in terms of the bosonic operator (25) as

$$
\xi_{i}^{\mathrm{N}}(\mathbf{x}, \lambda ; t)=-\overline{\bar{g}}_{\alpha \beta}^{-1} \int_{0}^{\infty} d \omega \sqrt{\frac{\hbar \operatorname{Im}[\gamma(\omega)]}{\pi}}\left(\left(\dot{\bar{a}}_{j \beta}-\imath \omega \overline{\bar{a}}_{j \beta}\right) b_{j}(\omega, 0) e^{-\imath \omega t}+\text { h.c. }\right) .
$$

From these results, the close formal analogy between the classical and quantum treatments of the dissipative Higgs model can be seen. We can easily show that in the classical limit, $\hbar \rightarrow 0$, Eq. (34) properly reduces to the classical analogue one (17), in the sense that in this limit all commutators vanish and the equation of motion becomes an equation for c-numbers driven by a noise force function. 


\subsection{Transition rates}

In this section, as an application of the presented approach, we investigate briefly the question of transition probabilities per unit time between two arbitrary states. For this purpose, we consider only the first order processes and write the Hamiltonian (23), up to first order of approximation in $\lambda$, as

$$
\mathcal{H}=\mathcal{H}_{0}+\mathcal{H}_{\text {int }},
$$

where

$$
\mathcal{H}_{0}=\mathcal{H}_{\text {Higgs }}(\mathbf{x}, \mathbf{p} ; \lambda)+\mathcal{H}_{\mathrm{r}}
$$

and

$$
\mathcal{H}_{\text {int }}=\int_{0}^{\infty} d \omega \sqrt{\frac{\hbar \operatorname{Im}[\gamma(\omega)]}{\pi}}\left\{\left(p_{\beta} \overline{\bar{a}}_{j \beta}+\lambda p_{\beta} \overline{\bar{a}}_{j \beta} x_{\alpha} x_{\alpha}\right) b_{j}(\omega, t)+\text { h.c. }\right\} .
$$

In the Hamiltonian (36), the term $R^{2}$ has been omitted since it can give rise to the transitions only in second or higher orders while we study here the first order processes in which the number of photons changes by \pm 1 . In addition, for simplicity, we restrict our concentration to a sufficiently small curvature. Therefore, the terms including the second and higher power of the curvature $\lambda$ have been ignored too. We calculate the transition probability at time $t$ from an initial state $\left|m, M_{j}(\omega)\right\rangle$ of $\mathcal{H}_{0}$ to a final state $\left|n, M_{j}(\omega)+1\right\rangle$ by treating $\mathcal{H}_{\text {int }}$ as a perturbation,

$$
\begin{aligned}
& P_{m, M_{j}(\omega) \rightarrow n \neq m, M_{j}(\omega)-1}= \\
& \frac{1}{\hbar^{2}}\left|\int_{0}^{t} d t^{\prime}\left\langle n, M_{j}(\omega)-1\left|e^{\frac{\imath}{\hbar} \mathcal{H}_{0} t^{\prime}} \mathcal{H}_{\text {int }} e^{-\frac{2}{\hbar} \mathcal{H}_{0} t^{\prime}}\right| m, M_{j}(\omega)\right\rangle\right|^{2} \\
& =\frac{1}{8 \hbar \omega}\left|\int_{0}^{t} d t^{\prime} f(\omega) \sqrt{M_{j}(\omega)} e^{\imath\left(\omega_{n m}-\omega\right) t^{\prime}}\left(V_{j, n m}+\lambda V_{j, n m}^{\prime}\right)\right|^{2},
\end{aligned}
$$

where $\omega_{n m}=\left(E_{n}-E_{m}\right) / \hbar$,

$$
V_{j, n m}=\langle n|\left(p_{\beta} \overline{\bar{a}}_{j \beta}+\text { h.c. }\right)|m\rangle,
$$

and

$$
V_{j, n m}^{\prime}=\langle n|\left(p_{\beta} \overline{\bar{a}}_{j \beta} x_{\alpha} x_{\alpha}+\text { h.c. }\right)|m\rangle .
$$


If $V_{j, n m}$ and $V_{j, n m}^{\prime}$ are explicitly time-independent, that is the case here, we may carry out the integral of Eq. (39) and obtain

$$
\begin{aligned}
P_{m, M_{j}(\omega) \rightarrow n \neq m, M_{j}(\omega)-1}= & \frac{\operatorname{Im}[\gamma(\omega)] M_{j}(\omega) \sin ^{2}\left[\left(\omega_{n m}-\omega\right) t / 2\right]}{4 \pi \hbar\left[\left(\omega_{n m}-\omega\right) t / 2\right]^{2}} \\
& \times\left|V_{j, n m}+\lambda V_{j, n m}^{\prime}\right|^{2} .
\end{aligned}
$$

As is known, the factor $\frac{\sin ^{2}\left[\left(\omega_{n m}-\omega\right) t / 2\right]}{\left[\left(\omega_{n m}-\omega\right) t / 2\right]^{2}}$ in Eq. (42) is a very strongly peaked function of $\omega_{n m}$. At $\omega_{n m}=\omega$ its amplitude increases as $t$ and decreases to zero when $\omega_{n m}-\omega=2 \pi / t$. The probability that $V_{j, n m}$ and $V^{\prime}{ }_{j, n m}$ induce the main system to make a transition between state $|n\rangle$ and $|m\rangle$ is thus very small unless energy is conserved between the initial and final states. We may therefore replace this highly packed factor by the Dirac function $2 \pi t \delta\left(\omega_{n m}-\omega\right)$ in Eq. (42). Thus, the transition probability per unit time for the absorption of a phonon is given by

$\Gamma_{m, M_{j}(\omega) \rightarrow n \neq m, M_{j}(\omega)-1}=\frac{\operatorname{Im}[\gamma(\omega)]}{2 \hbar} M_{j}(\omega)\left|V_{j, n m}+\lambda V^{\prime}{ }_{j, n m}\right|^{2} \delta\left(\omega_{n m}-\omega\right),($

which is the desired golden rule.

In a similar way, the transition probability per unit time from an initial state $\left|m, M_{j}(\omega)\right\rangle$ of $\mathcal{H}_{0}$ to a final state $\left|n, M_{j}(\omega)+1\right\rangle$ is obtained as

$\Gamma_{m, M_{j}(\omega) \rightarrow n \neq m, M_{j}(\omega)+1}=\frac{\operatorname{Im}[\gamma(\omega)]}{2 \hbar}\left[M_{j}(\omega)+1\right]\left|V_{j, n m}+\lambda V_{j, n m}^{\prime}\right|^{2} \delta\left(\omega_{n m}+\omega\right)$.

This equation is the probability of the main system to decay spontaneously when no radiation was initially present.

To summarize, we obtain the appreciable transition probability only if $E_{n} \simeq E_{m}-\hbar \omega$ (stimulated emission) or $E_{n} \simeq E_{m}+\hbar \omega$ (absorption). The particle on the sphere may, therefore, be changed from lower (upper) states to upper (lower) ones by using suitable frequencies of the reservoir's oscillators.

\section{$4 \quad$ Summary and concluding remarks}

In this paper, based on the Lagrangian scheme, the classical and the quantummechanic treatment of the damped particle on a sphere under the action of a conservative central force is investigated. In this approach, the dissipation and the fluctuation effects are introduced to our formalism by interacting the 
reservoir containing of continuum of three dimensional harmonic oscillators with the main system. As a direct consequence of this interaction, the explicit expressions for the projected susceptibility and quantum noise in terms of the coupling function and the metric of the physical system are obtained. It is shown that for the damped particle in the tangent plane, though the reservoir is assumed to be isotropic on the spherical surface, the projected susceptibility displays anisotropic character.

In Heisenberg picture, the Hamiltonian and in turn the quantum Langevin equation is derived. Of course, this Hamiltonian appears as a minimal coupling form somehow that the conjugate momentum of the particle coupled to the field operator $\mathbf{R}$. It is seen that the field operator $\mathbf{R}$ is expressed in terms of the infinite bosonic field operators. The noise operator in this approach are expressed in terms of these bosonic operators at the initial time and the metric of the physical system. It is stressed that the formalism tends to the normal Higgs model when the dissipative effects is neglected.

By using the perturbation theory, due to the dissipative effects, the transition rate between energy levels is calculated. It is shown that appreciable probabilities for transition are possible only if the transition and reservoir's oscillators frequencies are nearly on resonance, e.g., if, $E_{n} \simeq E_{m}-\hbar \omega$ (stimulated emission) or by $E_{n} \simeq E_{m}+\hbar \omega$ (absorption). Therefore, the particle on the sphere may be changed from lower(upper) states to upper(lower) ones by selecting suitable frequencies of the reservoir's oscillators.

\section{References}

[1] P.W. Higgs, J. Phys. A: Math. Gen. 12 (1979) 309.

[2] H.I. Leemon, J. Phys. A: Math. Gen. 12 (1979) 489.

[3] A. Mahdifar , R. Roknizadeh and M.H. Naderi, J. Phys. A: Math. Gen. 39 (2006) 7003.

[4] A.I. Solomon, Phys. Lett. A 196 (1994) 29; J. Katriel and A.I. Solomon, Phys. Rev. A 49 (1994) 5149; P. Shanta, S. Chaturvdi, V. Srinivasan and R. Jagannathan, J. Phys. A: Math. Gen. 27 (1994) 6433.

[5] A. Mahdifar, W. Vogel, T. Richter, R. Roknizadeh and M.H. Naderi, Phys. Rev. A 78 (2008) 063814. 
[6] A. Mahdifar, R. Roknizadeh and M.H. Naderi, Int. J. Geom. Methods Mod. Phys. 9 (2012) 1250009.

[7] A. Mahdifar, Int. J. Geom. Methods Mod. Phys. 10 (2013) 1350028.

[8] A. Mahdifar, B. Mirza, R. Roknizadeh, J. Phys. A: Math. Gen. 45(46) (2012) 465301.

[9] G.W. Ford, M. Kac, and P. Mazur, J. Math. Phys. 6 (1965) 504; G.W. Ford, J.T. Lewis, and R.F. OConnell, Phys. Rev. A 37 (1988) 4419.

[10] H. Mori, Progr. Theor. Phys. 33 (1965) 423.

[11] A.O. Caldeira, A.J. Leggett, Physica A 121 (1983) 587; A.O. Caldeira, A.J. Leggett, Ann. Phys. 149 (1983) 374.

[12] A.J. Leggett, et al, Rev. Mod. Phys. 59 (1987) 1.

[13] J. Schwinger, J. Math. Phys. 2 (1961) 407.

[14] L.V. Keldysh, Sov. Phys. JETP 20 (1965) 1018.

[15] R. Feynman, F. Vernon, Ann. Phys. 24 (1963) 118.

[16] H. Grabert, P. Schramm, G.L. Ingold, Phys. Rep. 168 (1988) 115.

[17] B.L. Hu, J.P. Paz, Y. Zhang, Phys. Rev. D 45,(1992) 2843.

[18] U. Weiss, Quantum Dissipative Systems, World Scientific Publishing, Singapore, 2008.

[19] E. Amooghorban and F. Kheirandish, Int. J. Theor. Phys. 53 (2014) 2593.

[20] F. Kheirandish and E. Amooghorban, Phys. Rev. A 82 (2010) 042901; E. Amooghorban, M. Wubs, N.A. Mortensen, and F. Kheirandish, Phys. Rev. A 84 (2011) 013806.

[21] M. Amooshahi, and E. Amooghorban, Ann. Phys. 325 (2010) 1976; F. Kheirandish, E. Amooghorban and M. Soltani, Phys. Rev. A 83 (2011) 032507;

[22] J.J. Hopfield, Phys. Rev. 112 (1958) 1555. 